\title{
Paternal obesity and its transgenerational effects on gastrointestinal function in male rat offspring
}

\author{
M.P.R. Machado ${ }^{1 \oplus}$, L.A. Gama ${ }^{2 \oplus}$, A.P.S. Beckmann ${ }^{2 \odot}$, A.T. Hauschildt' ${ }^{3 \oplus}$, D.J.R. Dall'Agnol ${ }^{4 \oplus}$, \\ J.R.A. Miranda ${ }^{1 \oplus}$, L.A. Corá ${ }^{5}$, and M.F. Américo ${ }^{20}$ \\ ${ }^{1}$ Instituto de Biociências, Universidade Estadual Paulista Júlio de Mesquita Filho, Botucatu, SP, Brasil \\ ${ }^{2}$ Instituto de Ciências Biológicas e da Saúde, Universidade Federal de Mato Grosso, Barra do Garças, MT, Brasil \\ ${ }^{3}$ Departamento de Fisiologia e Farmacologia, Universidade Federal do Ceará, Fortaleza, CE, Brasil \\ ${ }^{4}$ Faculdade de Ciências Agrárias, Biológicas, Engenharia e da Saúde, Universidade do Estado de Mato Grosso, \\ Tangará da Serra, MT, Brasil \\ ${ }^{5}$ Núcleo de Ciências Biológicas, NUCIB, Universidade Estadual de Ciências da Saúde de Alagoas (UNCISAL), \\ Maceió, AL, Brasil
}

\begin{abstract}
The interplay between obesity and gastrointestinal $(\mathrm{Gl})$ motility is contradictory, and the transgenerational influence on this parameter is unknown. We aimed to evaluate the Gl function in a model of paternal obesity and two subsequent generations of their male offspring. Newborn male rats were treated with monosodium glutamate (MSG) and composed the F1 generation, while control rats (CONT) received saline. At 90 days, male F1 were mated with non-obese females to obtain male offspring (F2), which later mated with non-obese females for obtaining male offspring of F3 generation. Lee Index analysis was adopted to set up the obesity groups. Alternating current biosusceptometry (ACB) technique was employed to calculate $\mathrm{Gl}$ transit parameters: mean gastric emptying time (MGET), mean cecum arrival time (MCAT), mean small intestinal transit time (MSITT), and gastric frequency and amplitude of contractions. Glucose, insulin, and leptin levels and duodenal morphometry were measured. F1 obese rats showed a decrease in the frequency and amplitude of gastric contractions, while obese rats from the F2 generation showed accelerated MGET and delayed MCAT and MSITT. Glucose and leptin levels were increased in F1 and F2 generations. Insulin levels decreased in F1, F2, and F3 generations. Duodenal morphometry was altered in all three generations. Obesity may have paternal transgenerational transmission, and it provoked disturbances in the gastrointestinal function of three generations.
\end{abstract}

Key words: Epigenetics; Gastric emptying; Intestinal transit; Monosodium glutamate

\section{Introduction}

Obesity is a multifactorial disease and has become a global pandemic. Over 650 million obese people $(1,2)$ face a complex interplay between biological, epigenetic, psychosocial, environmental, and industrial factors $(2,3)$.

Both non-genetic effects and genetic inheritance as well as epigenetic dysregulation can contribute to the predisposition to obesity (4). In this context, parental health or exposures can affect the lifetime health outcomes of offspring $(4,5)$. Since maternal effects have been the focus of the transgenerational transmission of metabolic disorders (5-9), the role of paternal obesity in offspring metabolic programming has been investigated but still not fully elucidated $(8,10)$.

Experimental models might contribute towards understanding the pathophysiological mechanisms and consequences concerning transgenerational obesity $(6,9)$.
When administered to neonatal rats, monosodium glutamate (MSG) is recognized to cause obesity and clinical manifestations of metabolic syndrome $(6,11)$. MSG provokes hypothalamic lesions resulting in several endocrine disturbances, stunted growth, and obesity $(7,12)$. MSGinduced maternal obesity results in spontaneously obese animals in the second generation (6).

Obesity is often associated with complications that affect cardiovascular, endocrine, and gastrointestinal (GI) systems $(2,13,14)$. GI motility, in particular, plays a very critical role in the digestion and absorption of nutrients (15), and also participates in the control of appetite and satiety (13). However, the relationship between obesity and $\mathrm{GI}$ motility is conflicting, and transgenerational effects have not been evaluated properly $(10,16)$. We hypothesized that obesity can alter parenteral $\mathrm{Gl}$ function and 
such effect can also manifest in the male offspring. The aim of this study was to evaluate the GI function in a model of paternal obesity and in two subsequent generations of their male offspring.

\section{Material and Methods}

\section{Experimental protocol}

All experiment procedures were approved by the Ethics Committee on Animal Research of the Federal University of Mato Grosso (protocol number 23108. 705702/13-9), following the 3Rs principle (Replacement, Reduction, and Refinement) of animal research. Wistar rats had free access to water and standard diet (Labina Presence ${ }^{\circledR}$, USA) and were kept in individual cages under controlled conditions of temperature $\left(22 \pm 1^{\circ} \mathrm{C}\right)$, humidity (50 $\pm 10 \%)$, and a 12-h light/dark cycle.

For obesity induction, male neonatal Wistar rats received $4 \mathrm{mg} / \mathrm{g}$ body weight subcutaneous (sc) injections of MSG (Sigma-Aldrich, USA) on days 2, 4, 6, 8, and 10 of postnatal life. Control Wistar rats received subcutaneous injections of saline solution $(0.9 \% \mathrm{NaCl})$ on the same days $(6,9)$. Both groups were weaned at 21 days of age and maintained in controlled conditions.

The Lee index was applied to all the male rats at 90 days of life. This index is defined as the cube root of body weight $(\mathrm{g}) \times 10 /$ naso-anal length $(\mathrm{cm})$ and values $>0.300$ are classified as obese $(6,9)$. All obese animals included in the MSG group were defined as F1 generation.

Obese rats of F1 generation were mated with nonobese adult female rats (90 days of age) and upon pregnancy detection (sperm-positive confirmation), pregnant rats were housed in individual cages. At 90 days of life, the male offspring from F1 were classified using the Lee index and established the F2 generation. Subsequently, the F2 generation rats, at 90 days of life, were mated with non-obese adults female rats (90 days of age), and their male offspring were also classified using the Lee index establishing the $\mathrm{F} 3$ generation.

The experimental groups were categorized as: Control group (CONT, n=14), MSG-induced obese rats ( $F 1, n=14)$, Obese offspring ( $F 2, n=14)$, and Grand offspring (F3, $n=14$ ). After assessing the Lee index, the animals from each generation had gastrointestinal motility, metabolic, and histological parameters evaluated and compared with the non-obese control group. All the variables were analyzed at the same age in all animals.

\section{Gastrointestinal motility}

GI motility was evaluated by the alternating current biosusceptometry (ACB) technique. ACB uses non-invasive and radiation-free sensors to record variations on the biomagnetic field generated by an ingested magnetic material. The intensity of the signal is proportional to the amount of magnetic material and depends on the distance to the sensor (17). To measure the $\mathrm{Gl}$ parameters (contractility and transit), all the animals fasted for $12 \mathrm{~h}$. From then on, they were fed with a magnetic meal $(1.6 \mathrm{~g}$ commercial chow plus $0.4 \mathrm{~g}$ ferrite powder) before the measurements. Ferrite $\left(\mathrm{MgZnFe}_{2} \mathrm{O}_{3}\right.$, Imag, Brazil) is an inert ferromagnetic material that is not absorbed by the $\mathrm{GI}$ tract and is used as a magnetic marker. The ACB sensor was used to monitor the magnetic signals on the abdominal surface at $15 \mathrm{~min}$ intervals for at least $6 \mathrm{~h}(18)$.

For gastric contractility records, all the animals fasted for $12 \mathrm{~h}$. Afterward, they ingested the magnetic meal described above and were anesthetized with $100 \mathrm{mg} / \mathrm{kg}$ ketamine (Cetamin ${ }^{\circledR}$, Syntec, Brazil) plus $2.5 \mathrm{mg} / \mathrm{kg}$ acepromazine (Acepran ${ }^{\circledR}$, Vetnil, Brazil), intraperitoneally. The animals were laid in a supine position and the ACB sensor was placed on the animal's abdominal surface in the corresponding anatomic region of the stomach. Magnetic signals were recorded continuously for $30 \mathrm{~min}$ at a sampling rate of $20 \mathrm{~Hz}$, using a multichannel recorder (MP100 System; BIOPAC, USA) (17).

\section{Magnetic data analysis}

To quantify the $\mathrm{Gl}$ transit, the statistical moment was applied (19). The temporal average determined by magnetic intensity curves normalized by the area under the curve was employed to quantify the following parameters: mean gastric emptying time (MGET), which was defined as the time $t$ ( $\mathrm{min}$ ) when a mean amount of magnetic meal was emptied from the stomach; mean cecum arrival time (MCAT), defined as the time $t$ ( $\min$ ) when an increase occurred in the mean amount of magnetic meal that arrived in the cecum; and mean small intestinal transit time (MSITT), which was quantified by the difference between MCAT and MGET (18).

To quantify the gastric contractility parameters, all magnetic signals were analyzed in MatLab (Mathworks, Inc., USA) by visual inspection and thereafter using bi-directional Butterworth band-pass filters by Fast Fourier Transform (FFT). The highest frequency peak for each FFT was defined as the gastric dominant frequency and the smallest, as the signal noise. The amplitude of contraction $(A)$ was calculated by the relationship between the power of gastric peak $(P)$ and power of noise peak $\left(P^{\prime}\right)$ and is expressed in decibels $(d B)$ as follows: $A=10$ $\log _{10}\left(P / P^{\prime}\right)(20)$.

\section{Serum and tissue sample collection}

At the end of the $\mathrm{Gl}$ motility evaluation, the animals were sacrificed at 120 days of life by anesthetic overdose through an intraperitoneal injection of ketamine $(240 \mathrm{mg} / \mathrm{kg}$, Cetamin $^{\circledR}$, Syntec) plus xylazine hydrochloride $(45 \mathrm{mg} / \mathrm{kg}$, Dopaser $^{\mathbb{R}}$, HertapeCalier, Brazil), followed by decapitation. Blood serum was sampled and stored under $-80^{\circ} \mathrm{C}$ refrigeration until further assays. 


\section{Histological preparations and measurements}

Duodenum tissue samples were carefully collected and fixed with Metacarn (60\% methanol, 30\% chloroform, and $10 \%$ glacial acetic acid), dehydrated with serial alcohol, diaphanized in xylol, and embedded in paraffin. Histological cuts were performed with Micron HM 355S Automatic Microtomer (Thermo Scientific, Germany) with $4-\mu \mathrm{m}$ thickness. The sections were stained with hematoxylin-eosin (H\&E) and the images were captured on an optical microscope (Zeiss, Germany) coupled to a highresolution camera (AxioCam ERc5s, Zeiss). Fifteen images per animal were randomly selected for morphometric analysis of the thickness of circular muscle and longitudinal muscle layers, villus height, and crypt depth using Image J software (NIH, USA). The villus height:crypt depth ratio was calculated (20).

\section{Glucose, insulin, and leptin serum quantifications}

Glucose levels were measured immediately after serum collection by Glucose Liquiform enzymatic kit (Labtest, Brazil). ELISA immunoassays were used for leptin (Rat Leptin kit, Invitrogen, USA) and insulin (Rat Insulin kit, Elabscience Biotechnology Co. Ltd., USA) serum concentration analyses according to manufacturers' instructions.

\section{Statistical analysis}

Kolmogorov-Smirnov test was employed to assess the normality of data. The data are reported as means $\pm S D$ with a limit of statistical significance up to $5 \%(P<0.05)$. Analysis of variance (ANOVA) followed by Tukey's post hoc test was performed for the multiple comparisons.

\section{Results}

Table 1 shows the frequency of obesity, Lee index, and body weight for all groups studied. From the MSG-induced obese rats (F1), $73.3 \%$ of offspring in the $F 2$ generation, and $7.0 \%$ of grand offspring in the $\mathrm{F} 3$ generation were obese, according to the parameters evaluated. Considering these results, only obese animals from F1 and F2 generations, and non-obese animals from F3 generation continued in the study because they were the large majority in each group. Furthermore, body weight was significantly higher for F1 and F2 groups (obese generations) compared with CONT group, whereas body weight of F3 generation (non-obese) was not different from the CONT group.

Figure 1 shows the glucose, insulin, and leptin levels for F1, F2, and F3 generations. Glucose levels were higher for the obese groups, MSG-induced obese rats (F1) and F2 generation offspring compared with CONT and F3 $(P<0.001)$. In addition, F2 showed a slightly lower glucose level compared with $\mathrm{F} 1(\mathrm{P}<0.05)$. No differences were found between $\mathrm{F} 3$ generation and CONT groups. In F1 obese and F2 obese groups, insulin levels were lower compared to CONT $(\mathrm{P}<0.05)$. Insulin levels for F3 were

Table 1. Frequency of obesity, Lee index, and body weight of Control (CONT), monosodium glutamateinduced obese rats (F1), obese offspring (F2), and grand offspring (F3) groups.

\begin{tabular}{lcccc}
\hline & CONT & F1 & F2 & F3 \\
\hline Obesity in the generations & $0 \%(n=14)$ & $100 \%(n=14)$ & $77.8 \%(14 / 18)$ & $7.0 \%(1 / 15)$ \\
Obesity in the experimental groups included in analysis & $0 \%(n=14)$ & $100 \%(n=14)$ & $100 \%(n=14)$ & $0 \%(n=14)$ \\
Lee index & $<0.300$ & $0.313 \pm 0.010$ & $0.325 \pm 0.020$ & $<0.300$ \\
Body weight $(\mathrm{g})$ & $296.1 \pm 23.1$ & $341.8 \pm 20.7^{*}$ & $336.2 \pm 60.8^{*}$ & $329.8 \pm 26.4$ \\
\hline
\end{tabular}

Only the 14 obese animals from F2 and the 14 non-obese animals in F3 were included in the analysis. Data are reported as means $\pm \mathrm{SD}$. ${ }^{*} \mathrm{P}<0.05$ compared with CONT (ANOVA followed by Tukey's post hoc).
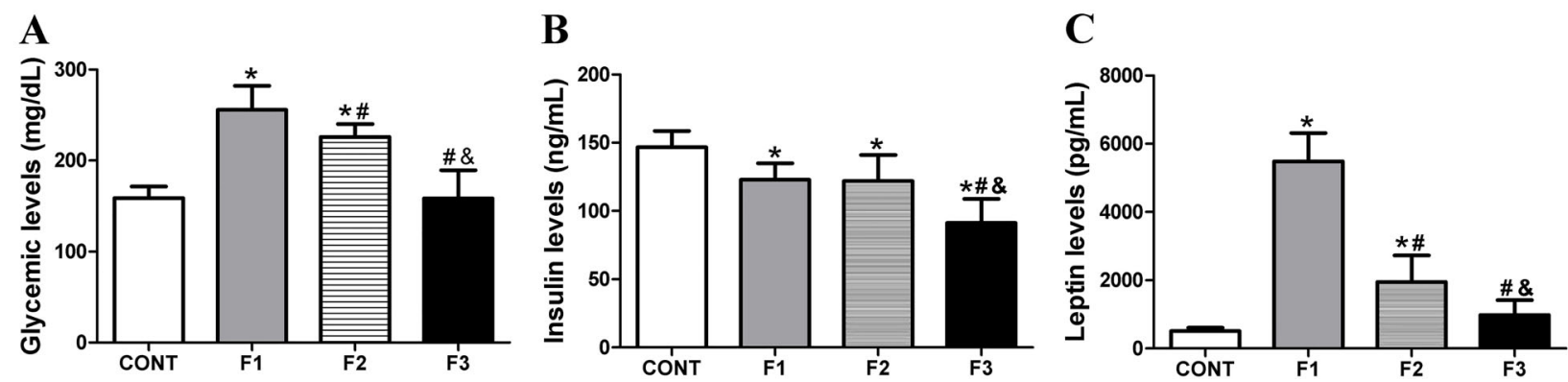

Figure 1. Glucose (A), insulin (B), and leptin (C) levels for Control group (CONT), monosodium glutamate (MSG)-induced obese rats $(F 1)$, obese offspring (F2), and grand offspring (F3). Data are reported as means $\pm S D(n=14$ per group). * $P<0.05$ compared with CONT, ${ }^{\#} \mathrm{P}<0.05$ compared with $\mathrm{F} 1$, and ${ }^{\&} \mathrm{P}<0.05$ compared with F2 (ANOVA followed by Tukey's post hoc). 

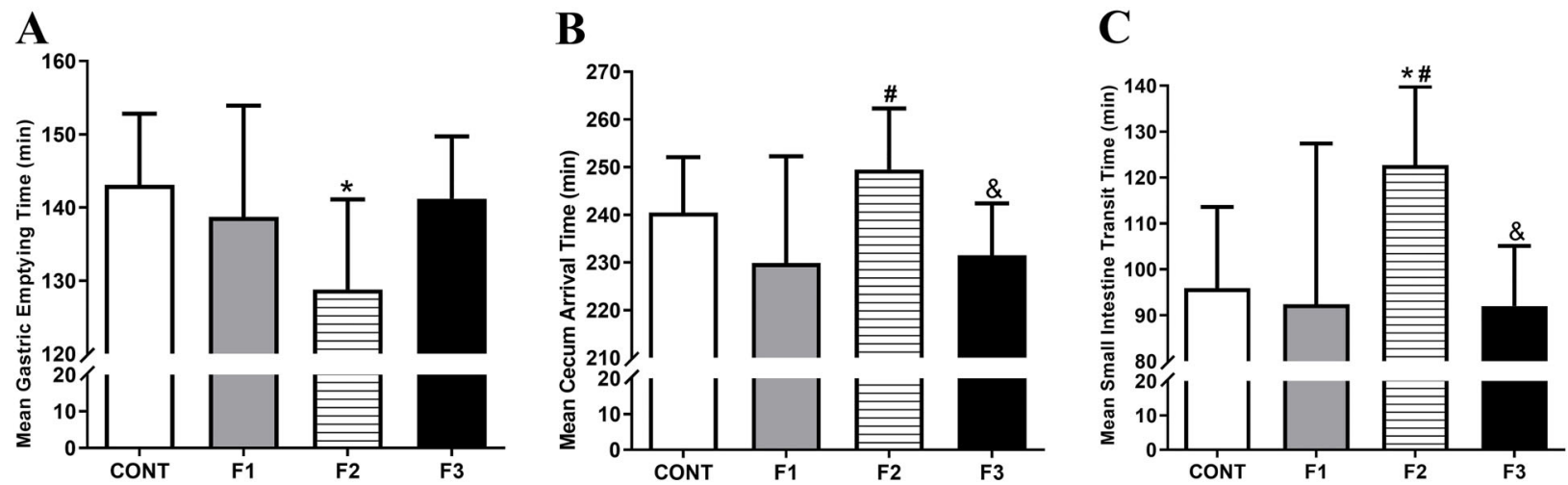

Figure 2. Gastrointestinal transit parameters evaluated for the Control group (CONT), monosodium glutamate (MSG)-induced obese rats (F1), obese offspring (F2), and grand offspring (F3). Mean gastric emptying time (MGET, A), mean cecum arrival time (B); mean small intestine transit time $(\mathbf{C})$. Data are reported as means $\pm S D\left(n=14\right.$ per group). ${ }^{*} P<0.05$ compared with CONT, ${ }^{\#} P<0.05$ compared with $\mathrm{F} 1$, and ${ }^{\circledR} \mathrm{P}<0.05$ compared with F2 (ANOVA followed by Tukey's post hoc).

significantly lower compared with CONT $(\mathrm{P}<0.01)$, F1 $(\mathrm{P}<0.05)$, and $\mathrm{F} 2(\mathrm{P}<0.01)$ generations. Leptin levels were increased in the F1 and F2 obese groups compared to CONT ( $P<0.001$ and $P<0.01$, respectively). The $F 1$ group presented higher leptin levels than the $\mathrm{F} 2$ generation $(P<0.001)$. Rats in the F3 generation had no difference in leptin levels compared to the CONT group.

The GI transit parameters evaluated in all groups are shown in Figure 2. The F2 obese group had accelerated gastric emptying compared with the CONT group $(P<0.05)$. No difference was found among F1, F2, and $\mathrm{F} 3$ groups for gastric emptying time. Cecum arrival in F2 was delayed compared with $F 1(P<0.05)$ and $F 3$ groups $(P<0.05)$, although it was not different from the CONT group. The small intestine transit time in the F2 group was delayed compared with CONT, F1, and F3 groups $(P<0.05)$.

Gastric contractility parameters evaluated in all groups are shown in Figure 3. In the F1 group, the frequency of gastric contractions was reduced compared with CONT, $F 2$, and F3 groups $(P<0.01)$. No significant difference was found between the F2 and F3 generations compared to the CONT group. The amplitude of gastric contractions was similar between the CONT and F3 groups (41.30 \pm 4.78 $\mathrm{dB}$ and $43.10 \pm 8.10 \mathrm{~dB}$ ), and both were significantly higher compared with the F1 generation $(33.65 \pm 3.43 \mathrm{~dB} ; \mathrm{P}<0.05$ and $\mathrm{P}<0.01$, respectively).

Representative H\&E-stained duodenum tissue and the histological and morphometric analyses are presented in Figure 4. The thickness of the circular muscle layer for the F1 and F3 groups was thinner compared with the CONT group $(P<0.01)$, and in $F 1$ it was significantly thinner than F2 $(\mathrm{P}<0.01)$. No significant difference was observed in the F2 group compared with the CONT group. In the longitudinal muscle layer, the thickness was even thinner in $\mathrm{F} 1, \mathrm{~F} 2$, and $\mathrm{F} 3$ groups compared with CONT $(\mathrm{P}<0.01)$. All the generations presented both lower crypt depth and
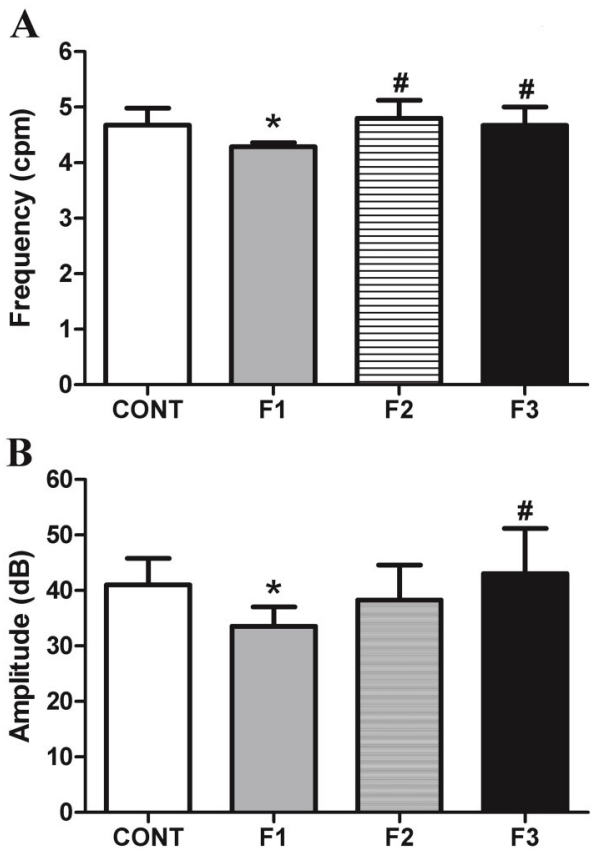

Figure 3. Gastric contractility parameters calculated for the Control group (CONT), monosodium glutamate (MSG)-induced obese rats (F1), obese offspring (F2), and grand offspring (F3). Frequency in contractions per min (cpm) (A) and amplitude (B) of gastric contractions. Data are reported as means $\pm S D(n=14$ per group). ${ }^{*} \mathrm{P}<0.05$ compared with $\mathrm{CONT}$, ${ }^{*} \mathrm{P}<0.05$ compared with F1 (ANOVA followed by Tukey's post hoc).

villus height compared with CONT $(P<0.001)$. The $F 2$ group presented greater villus height compared with the $F 1$ and $F 3$ groups $(P<0.01)$. In this regard, villus height to crypt depth ratios in F1 and F3 groups were smaller compared with CONT $(P<0.01)$. The $F 2$ generation presented a higher villus height to crypt depth ratio 

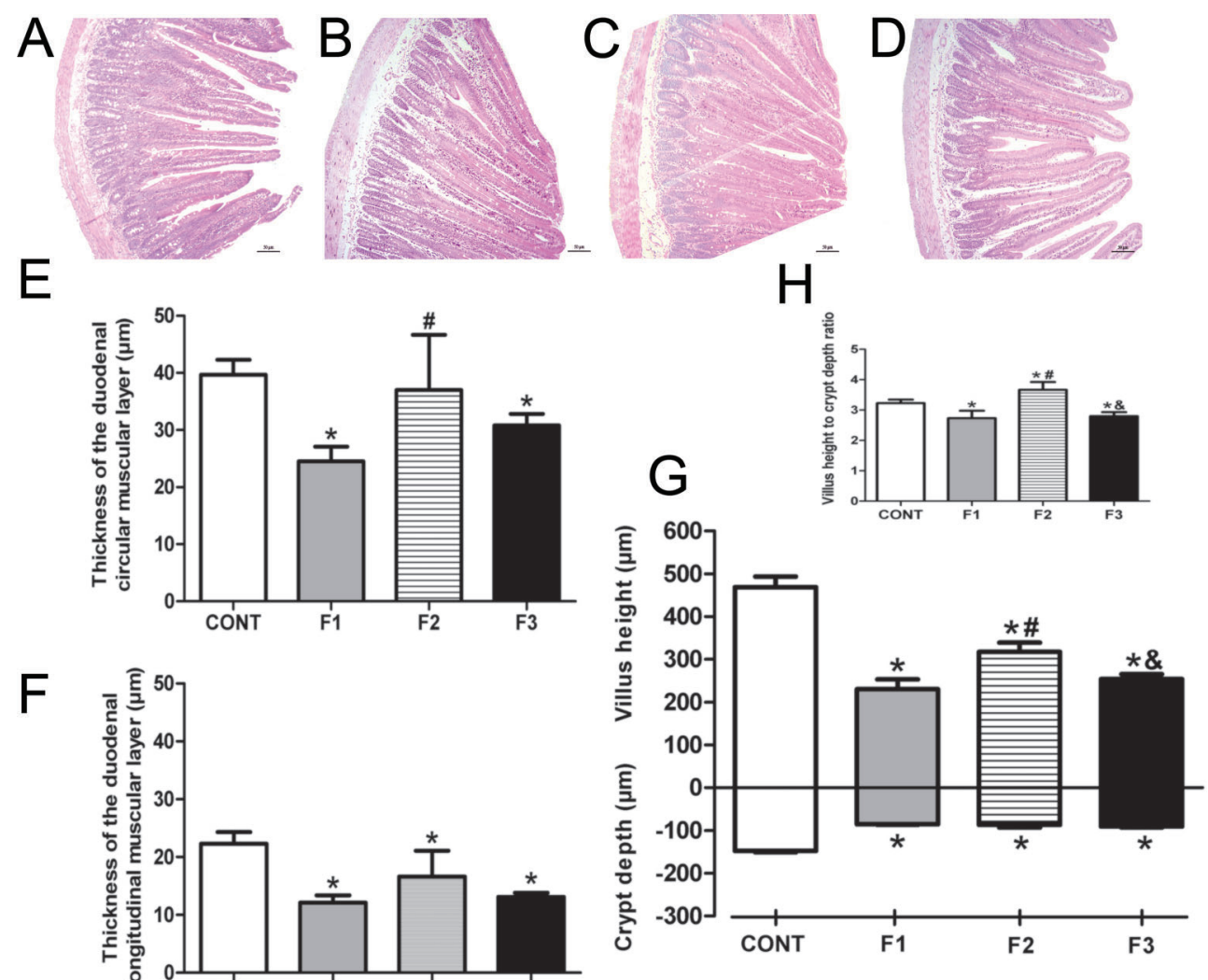

Figure 4. Histological photomicrographs of H\&E staining of the duodenum (A-D) of Control (CONT), monosodium glutamate (MSG)induced obese rats (F1), obese offspring (F2), and grand offspring (F3) $(\times 10$ objective, scale bar, $50 \mu \mathrm{m})$. Quantification of the thickness of the duodenal circular muscle layer $(\mathbf{E})$ and longitudinal muscle layer $(\mathbf{F})$, crypt depth, villus height $(\mathbf{G})$, and villus height to crypt depth ratio $(\mathbf{H})$. Data are reported as means $\pm S D\left(n=14\right.$ per group). ${ }^{*} \mathrm{P}<0.05$ compared with $C O N T$, ${ }^{*} P<0.05$ compared with $F 1$, and ${ }^{\&} \mathrm{P}<0.05$ compared with F2 (ANOVA followed by Tukey's post hoc).

compared with all groups $(\mathrm{P}<0.01$ compared to CONT and $\mathrm{P}<0.001$ compared to $\mathrm{F} 1$ and $\mathrm{F} 3$ ).

\section{Discussion}

In this study, we demonstrated for the first time that MSG-induced obesity led to paternally derived, transgenerational obesity, and gastrointestinal alterations in male rats. The MSG-induced obese rats (F1) and their obese offspring generation (F2) showed disorders in GI motility with increased glucose and leptin levels. No functional changes or obesity phenotype was observed in the grand offspring generation (F3). All generations had changes in the muscular layers and duodenal mucosa, strengthening the extent of the disturbances driven by obesity.

Body weight was significantly higher for the F1 and F2 generations (obese rats) compared to the control group, whereas the F3 generations, characterized as non-obese, presented body weight that was not different from the control. The percentage of obesity was $100.0,77.8$, and $7.0 \%$ for the F1, F2, and F3 generations, respectively. This pattern indicated that MSG-induced obesity leads to paternally derived obesity in the subsequent generation (F2), with minor effects in the grand offspring generation (F3). Studies have shown that neonatal administration of MSG sets a precedent for the development of obesity due to the hypothalamic lesion resulting in a Lee Index with values higher than 0.300 , typical physical characteristics, and delay in growth (12).

There are important interactions among adipose tissue, gut hormones, central nervous system, and GI tract in the development and maintenance of obesity $(14,21)$. The investigation of the relationship between obesity and GI motility remains inconsistent and scarce, despite that it could represent an opportunity for novel therapeutic approaches for the treatment of obesity or other $\mathrm{Gl}$ diseases $(13,14)$.

Gastric emptying plays an important role in the intestinal exposure to nutrients and satiety $(22,23)$, and it is closely related to glycemic control since it acts as a major determinant of postprandial glycemic regulation (24). In our study, both the F1 and F2 generations had 
changes in gastric motility and, in parallel, their glycemic levels were higher compared to the CONT and F3 groups. Our group has already demonstrated the relationship between these variables in mildly diabetic rats (25). The severity of gastric dysmotility and gastric repercussions seems to be inversely proportional to glycemic control (26).

Small bowel transit regulates nutrient absorption and seems to be related to the development of obesity (23). In our study, the F2 generation had a delayed MCAT and MSITT compared to all groups, which reinforced that some programmed effects can be transmitted to subsequent generations (27). As the absorption of nutrients from the small intestine is based on the efficiency of both digestive and epithelial transport mechanisms and the area of the intestinal mucosa, it is supposed that a delay in the intestinal transit may increase absorption and weight gain $(15,23)$. Other studies have shown that small intestinal transit $(13,21)$ and intestinal myoelectrical activity (13) were not affected by obesity induced by a high-fat diet (HFD).

Such conflicting findings regarding GI motility reported in obesity may be justified by different methodologies, analyses of the experimental data, and different species and/or strains used as animal models $(13,28)$. Although several methods are available to evaluate GI motility, most of them are invasive or restricted by ethical issues (29). ACB is a magnetic technique validated to evaluate $\mathrm{GI}$ motor functions in rodents repeatedly $(17,18,20)$. It is noninvasive and allows recording the GI motility physiologically in real time, without interfering with the system itself.

Despite motility changes, the gut is a target for histologic changes that need to be evaluated to handle the impact of the disease on different segments. In our study, the thickness of the longitudinal muscle layer in the duodenum was reduced in all generations; however, the circular muscle was reduced only in the F1 generation. Another study also observed a decrease of the small intestine wall thickness in MSG-induced obese rats, but subsequent generations were not evaluated (30).

Muscular layers offer different contributions to the intestinal motor patterns: the circular layer acts on the mixing motions of the luminal content, whereas the propulsion activity is triggered by the longitudinal layer (31). Based on these findings, we assume that thinner muscular layers could have slowed the intestinal transit in the F2 group. In addition, disorders on the myenteric plexus may also contribute to the damage on GI transit, since it is located between the longitudinal and circular layers of the muscular layer (32).

In duodenal mucosa, the height of the villi and the depth of the crypt as well as the villus:crypt ratio of the F1 and F3 generations were reduced. In the F2 group, there was a higher villus:crypt ratio compared with CONT, despite the height of the villi and depth of crypt being reduced. The small intestine absorptive function also depends on the villus distribution, and enterocyte and crypt integrity (33). Hence, while such a function may be compromised in F1 and F3, the greater villus height in F2 may have been an adaptive morphometric characteristic of this spontaneous obese generation to increase the intestinal absorptive area. The ileum of the MSG-induced obese mice had lower expression levels of the junction proteins increasing the intestinal permeability, suggesting that the damage of the intestinal barrier induces systemic inflammation (34).

Peptides secreted by adipocytes can modulate $\mathrm{GI}$ motility by acting on the central and enteric nervous system (14). Leptin is produced by adipose tissue and exerts its primary effect on the hypothalamic nuclei to regulate food intake and body weight (35). In addition, leptin seems to influence gastric emptying and small intestine motility $(21,35)$. Obese rats from F1 and F2 generations had higher leptin levels compared to CONT and F3 groups. Specifically, in the F1 generation, leptin concentrations were higher compared to F2. There was no difference between leptin levels from F3 and CONT groups. Thus, we believe that this may be one of the factors involved in the changes in GI motility that was observed in obese generations.

In our study, a reduction in the serum insulin concentrations in the F1, F2, and F3 generations compared to CONT was observed. Insulin secretion dysfunction seems to be a more permanent change across generations (7) and could be a characteristic inherited from an obese father. In female offspring from a father submitted to the HFD with normal adiposity, $\beta$-cell alterations with impaired insulin secretion and glucose tolerance have been observed, showing the intergenerational transmission of the metabolic sequel from father to offspring (36).

It is important to investigate the paternal implications of the onset of obesity and other metabolic disorders in offspring. MSG has shown toxic effects on several systems $(11,37)$, and offspring from MSG-induced obese rats can be used as a model for obesity studies since it impacts on offspring metabolism and body weight and can be compared to other models $(6,37)$.

In humans, overweight parents are considered as a risk factor for offspring overweight/obesity (38). Children from obese parents have an increased risk for obesity, reaching $80 \%$ when both parents are obese, $40 \%$ when only one parent is obese, and $10 \%$ when none is obese (39). In experimental studies, $80 \%$ of offspring rats from obese females were also obese (6). In our study, the F2 generation also showed a similar obese percentage in males, corroborating the strong paternal role in the transmission of obesity as pointed out in human studies (40). Thus, our data showed that the father can onset the intergenerational transmission of obesity induced or inherited, and this condition can also affect the gastrointestinal function. Furthermore, our study can contribute to new approaches in the field of developmental programming by 
considering the influence of paternal obesity and its transgenerational effects on offspring health.

In conclusion, MSG-induced obese rats and their obese offspring generations had disorders in the gastrointestinal function associated with increased levels of glucose and leptin parallel to the reduction of insulin levels. From the third generation, $\mathrm{Gl}$ variables were similar to control animals, despite morphometry being altered in all three generations. Our data added new insights about the physiology of obesity in rats and

\section{References}

1. Upadhyay J, Farr O, Perakakis N, Ghaly W, Mantzoros C. Obesity as a disease. Med Clin North Am 2018; 102: 13-33, doi: 10.1016/j.mcna.2017.08.004.

2. Lee MK, Blumberg B. Transgenerational effects of obesogens. Basic Clin Pharmacol Toxicol 2019; 125: 44-57, doi: 10.1111/bcpt.13214.

3. Ralston J, Brinsden H, Buse K, Candeias V, Caterson I, Hassell T, et al. Time for a new obesity narrative. Lancet 2018; 392: 1384-1386, doi: 10.1016/S0140-6736(18)32537-6.

4. Bays H, Scinta W. Adiposopathy and epigenetics: an introduction to obesity as a transgenerational disease. Curr Med Res Opin 2015; 31: 2059-2069, doi: 10.1185/03007995. 2015.1087983.

5. Vickers $\mathrm{MH}$. Developmental programming and transgenerational transmission of obesity. Ann Nutr Metab 2014; 64: 26-34, doi: 10.1159/000360506.

6. Campos KE, Sinzato YK, Pimenta WP, Rudge MV, Damasceno DC. Effect of maternal obesity on diabetes development in adult rat offspring. Life Sci 2007; 81: 14731478, doi: 10.1016/j.Ifs.2007.09.016.

7. Miranda RA, Agostinho AR, Trevenzoli IH, Barella LF, Franco CCS, Trombini $A B$, et al. Insulin oversecretion in MSG-obese rats is related to alterations in cholinergic muscarinic receptor subtypes in pancreatic islets. Cell Physiol Biochem 2014; 33: 1075-1086, doi: 10.1159/000358677.

8. Hur SSJ, Cropley JE, Suter CM. Paternal epigenetic programming: evolving metabolic disease risk. $J$ Mol Endocrinol 2017; 58: R159-R168, doi: 10.1530/JME-16-0236.

9. Soares TS, Andreolla AP, Miranda CA, Klöppel E, Rodrigues LS, Moraes-Souza RQ, et al. Effect of the induction of transgenerational obesity on maternal-fetal parameters. Syst Biol Reprod Med 2018; 64: 51-59, doi: 10.1080/ 19396368.2017.1410866.

10. Lecomte V, Maloney CA, Wang KW, Morris MJ. Effects of paternal obesity on growth and adiposity of male rat offspring. Am J Physiol Endocrinol Metab 2017; 312: E117-E125, doi: 10.1152/ajpendo.00262.2016.

11. Uti DE, Atangwho IJ, Eyong EU, Umoru GU, Egbung GE, Nna VU, et al. African walnuts attenuate ectopic fat accumulation and associated peroxidation and oxidative stress in monosodium glutamate-obese Wistar rats. Biomed Pharmacother 2020; 124: 109879, doi: 10.1016/j.biopha. 2020.109879.

12. Bautista RJH, Mahmoud AM, Königsberg M, Guerrero NELD. Obesity: pathophysiology, monosodium glutamateinduced model and anti-obesity medicinal plants. Biomed contributed to understanding the extent of the disturbances in gastrointestinal physiology provoked by a paternal cycle of obesity.

\section{Acknowledgments}

The authors would like to thank Me. Maysa Bruno de Lima for carrying out the initial experiments of this research and Dr. Kleber Eduardo de Campos for technical experimental support.
Pharmacother 2019; 111: 503-516, doi: 10.1016/j.biopha. 2018.12.108.

13. Wan $X$, Yin J, Chen J. Characteristics of intestinal myoelectrical and motor activities in diet-induced obese rats: obesity and motility. Dig Dis Sci 2019; 64: 1478-1485, doi: 10.1007/s10620-019-5458-4.

14. Emerenziani S, Guarino MPL, Asensio LMT, Altomare A, Ribolsi M, Balestrieri P, et al. Role of overweight and obesity in gastrointestinal disease. Nutrients 2020; 12: 111, doi: 10.3390/nu12010111.

15. Xing J, Chen JD. Alterations of gastrointestinal motility in obesity. Obes Res 2004; 12: 1723-1732, doi: 10.1038/oby. 2004.213.

16. Nguyen NQ, Debreceni TL, Burgess JE, Bellon M, Wishart $\mathrm{J}$, Standfield $\mathrm{S}$, et al. Impact of gastric emptying and small intestinal transit on blood glucose, intestinal hormones, glucose absorption in the morbidly obese. Int $J$ Obes (Lond) 2018; 42: 1556-1564, doi: 10.1038/s41366-018-0012-6.

17. Américo MF, Marques RG, Zandoná EA, Andreis $U$, Stelzer $\mathrm{M}$, Corá $\mathrm{LA}$, et al. Validation of $\mathrm{ACB}$ in vitro and in vivo as a biomagnetic method for measuring stomach contraction. Neurogastroenterol Motil 2010; 22: 1340-e374, doi: 10.1111/ j.1365-2982.2010.01582.x.

18. Quini CC, Américo MF, Corá LA, Calabresi MFF, Alvarez M, Oliveira RB, et al. Employment of a noninvasive magnetic method for evaluation of gastrointestinal transit in rats. $\mathrm{J} \mathrm{Biol}$ Eng 2012; 6: 6, doi: 10.1186/1754-1611-6-6.

19. Podczeck F, Newton JM, Yuen KH. The description of the gastrointestinal transit of pellets assessed by gamma scintigraphy using statistical moments. Pharm Res 1995; 12: 376-379, doi: 10.1023/A:1016200501563.

20. Gama LA, Machado MPR, Beckmann APS, Miranda JRA, Corá LA, Américo MF. Gastrointestinal motility and morphology in mice: Strain-dependent differences. Neurogastroenterol Motil 2020; 32: e13824, doi: 10.1111/nmo. 13824.

21. Li J, Ma W, Wang S. Slower gastric emptying in high-fat diet induced obese rats is associated with attenuated plasma ghrelin and elevated plasma leptin and cholecystokinin concentrations. Regul Pept 2011; 171: 53-57, doi: 10.1016/ j.regpep.2011.07.004.

22. Janssen $P$, Vanden Berghe $P$, Verschueren $S$, Lehmann A, Depoortere I, Tack J. Review article: the role of gastric motility in the control of food intake. Aliment Pharmacol Ther 2011; 33: 880-894, doi: 10.1111/j.1365-2036.2011. 04609.x. 
23. Sun $Y$, Chen J. Rimonabant, gastrointestinal motility and obesity. Curr Neuropharmacol 2012; 10: 212-218, doi: 10.2174/157015912803217297.

24. Phillips LK, Deane AM, Jones KL, Rayner CK, Horowitz M. Gastric emptying and glycaemia in health and diabetes mellitus. Nat Rev Endocrinol 2015; 11: 112-128, doi: 10.1038/ nrendo.2014.202.

25. Hauschildt AT, Corá LA, Volpato GT, Sinzato YK, Damasceno DC, Américo MF. Mild diabetes: long-term effects on gastric motility evaluated in rats. Int J Exp Pathol 2018; 99: 29-37, doi: 10.1111/iep.12262.

26. de Oliveira LCS, Telles PVN, Sousa JFR, Cavalcante AKM, Wong DVT, Lima-Junior RC, et al. Influence of the physical exercise on decrease in the gastric emptying and alter appetite and food behavior in rats dexamethasone-treatment. Physiol Behav 2019; 209: 112610, doi: 10.1016/j.physbeh. 2019.112610.

27. Portha B, Grandjean V, Movassat J. Mother or father: who is in the front line? mechanisms underlying the non-genomic transmission of obesity/diabetes via the maternal or the paternal line. Nutrients 2019; 11: 233, doi: 10.3390/nu110 20233.

28. Marques $C$, Meireles M, Norberto S, Leite J, Freitas J, Pestana D, et al. High-fat diet-induced obesity rat model: a comparison between Wistar and Sprague-Dawley Rat. Adipocyte 2015; 5: 11-21, doi: 10.1080/21623945.2015. 1061723.

29. Camilleri M, Linden DR. Measurement of gastrointestinal and colonic motor functions in humans and animals. Cell Mol Gastroenterol Hepatol 2016; 2: 412-428, doi: 10.1016/ j.jcmgh.2016.04.003.

30. Gomes JR, Freitas JR, Grassiolli S. Effects of physical exercise on the intestinal mucosa of rats submitted to a hypothalamic obesity condition. Anat Rec (Hoboken) 2016; 299: 1389-1396, doi: 10.1002/ar.23453.

31. Kumral D, Zfass AM. Gut movements: a review of the physiology of gastrointestinal transit. Dig Dis Sci 2018; 63: 2500-2506, doi: 10.1007/s10620-018-5259-1.
32. Lisboa MJS, Lima MFO, Stabille SR, Zanoni JN, Gagliardo $\mathrm{KM}$, Souto MS, et al. Supplementation action with ascorbic acid in the morphology of the muscular layer and reactive acetylcholinesterase neurons of ileum of $\mathrm{mdx}$ mice. Auton Neurosci 2017; 205: 57-66, doi: 10.1016/j.autneu.2017. 05.001.

33. Thomazini BF, Dolder MAH. Dose dependent treatment with isotretinoin induces more changes in the ileum than in the duodenum and jejunum in Wistar rats. Tissue Cell 2017; 49: 203-208, doi: 10.1016/j.tice.2017.03.003.

34. Nakadate K, Hirakawa T, Tanaka-Nakadate S. Small intestine barrier function failure induces systemic inflammation in monosodium glutamate-induced chronically obese mice. Appl Physiol Nutr Metab 2019; 44: 587-594, doi: 10.1139/apnm-2018-0560.

35. Goyal RK, Guo Y, Mashimo H. Advances in the physiology of gastric emptying. Neurogastroenterol Motil 2019; 31: e13546, doi: 10.1111/nmo.13546.

36. Ng SF, Lin RCY, Laybutt DR, Barres R, Owens JA, Morris MJ. Chronic high-fat diet in fathers programs $\beta$-cell dysfunction in female rat offspring. Nature 2010; 467: 963-966, doi: 10.1038/nature09491.

37. Afifi M, Abbas A. Monosodium glutamate versus diet induced obesity in pregnant rats and their offspring. Acta Physiol Hung 2011; 98: 177-188, doi: 10.1556/APhysiol.98. 2011.2.9.

38. Bahreynian M, Qorbani M, Khaniabadi BM, Motlagh ME, Safari $\mathrm{O}$, Asayesh $\mathrm{H}$, et al. Association between obesity and parental weight status in children and adolescents. $J$ Clin Res Pediatr Endocrinol 2017; 9: 111-117, doi: 10.4274/ jcrpe.3790.

39. Nguyen VT, Larson DE, Johnson RK, Goran MI. Fat intake and adiposity in children of lean and obese parents. Am J Clin Nutr 1996; 63: 507-513, doi: 10.1093/ajcn/ 63.4.507.

40. Soubry A. POHaD: why we should study future fathers. Environ Epigenet 2018; 4: dvy007, doi: 10.1093/eep/ dvy007. 